\title{
One Single Question Is not Sufficient to Identify Individuals With Electromagnetic Hypersensitivity
}

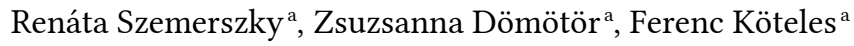 \\ [a] Institute of Health Promotion and Sport Sciences, ELTE Eötvös Loránd University, Budapest, Hungary.
}

Clinical Psychology in Europe, 2019, Vol. 1(4), Article e35668, https://doi.org/10.32872/cpe.v1i4.35668

Received: 2019-04-23 • Accepted: 2019-09-04 • Published (VoR): 2019-12-17

Handling Editor: Omer Van den Bergh, University of Leuven, Leuven, Belgium

Corresponding Author: Ferenc Köteles, Institute of Health Promotion and Sport Sciences, Eötvös Loránd University, Budapest, Bogdánfy Ödön u. 10., H-1117 Hungary. E-mail: koteles.ferenc@ppk.elte.hu

\begin{abstract}
Background: Idiopathic Environmental Intolerance attributed to Electromagnetic Fields (IEI-EMF) is a self-reported condition where non-specific symptoms are attributed to weak non-ionizing electromagnetic fields. Despite its expanding prevalence, there is no generally accepted diagnostic procedure or definition to identify patients with this condition, thus studies usually apply only one question as inclusion criterion. The aim of our study was to demonstrate the heterogeneity of a self-reported IEI-EMF group and to identify further self-report questions that could be applied as inclusion criteria.

Method: Cross-sectional on-line survey study was carried out with 473 participants (76.3\% women; age: $35.03 \pm 13.24$ yrs). Self-diagnosed IEI-EMF (as assessed with a yes-or-no question), frequency of EMF-related symptom and severity of the condition were assessed, as well as somatic symptom distress (Patient Health Questionnaire Somatic Symptom Severity Scale, PHQ-15).

Results: $72(15.2 \%)$ individuals labelled themselves as IEI-EMF, however only $61 \%$ of them remained in the IEI-EMF group after the use of three inclusion criteria instead of one. $21 \%$ of the individuals labelling themselves as IEI-EMF reported neither symptoms nor any negative impact on their daily life.

Conclusion: A minimum of two questions appear to be necessary as inclusion criteria for IEI-EMF in empirical research. Instead of the widely used yes-or-no question on accepting the IEI-EMF label, occurrence of symptoms attributed to EMF on a regular basis and at least a slight negative impact on daily life are required.
\end{abstract}

\section{Keywords}

electrohypersensitivity, symptoms, assessment, Idiopathic Environmental Intolerance Attributed to Electromagnetic Fields, IEI-EMF 


\section{Highlights}

- Electromagnetic hypersensitivity (IEI-EMF) is often assessed by one yes-or-no self-report question.

- This practice is inappropriate from a conceptual and methodological point of view.

- At least two questions, assessing frequency of symptoms and their impact, are needed.

According to the definition of the World Health Organization, the term Idiopathic Environmental Intolerance attributed to Electromagnetic Fields (IEI-EMF; formerly electromagnetic hypersensitivity) refers to "symptoms that are experienced in proximity to, or during the use of, electrical equipment, and that result in varying degrees of discomfort or ill health in the individual and that an individual attributes to activation of electrical equipment" (WHO, 2004, p. 2). Originally, IEI was defined along the following criteria: (1) an acquired disorder with multiple recurrent symptoms (2) that could be associated with diverse environmental factors tolerated by the majority of the population, and (3) cannot be explained by any other known disorder (medical or psychological) (Lessof, 1997; Staudenmayer, 2006).

Concerning IEI-EMF, however, some of the aforementioned criteria are unrealistic and practically irrelevant. First (Criterion 1), why does one want and how can one distinguish between acquired and inherited conditions in the modern era when the importance of epigenetics and environment-gene interactions is well described, and empirical findings concerning genetic factors (e.g. gene polimorphisms) behind environmental illnesses are accumulating (Berg et al., 2010; Caccamo et al., 2013; Cui et al., 2013; De Luca et al., 2015, 2014; McKeown-Eyssen et al., 2004; Schnakenberg et al., 2007)? Moreover, what is the difference between individuals with acquired and (partly) inherited IEI-EMF from a therapeutic point of view? Second (Criterion 3), although it is well documented that IEIEMF is often accompanied by co-morbid psychiatric disorders (e.g. depression, anxiety disorder) (Frick et al., 2005; Landgrebe et al., 2008; Meg Tseng, Lin, \& Cheng, 2011; Österberg, Persson, Karlson, Eek, \& Ørbæk, 2007; Rubin, Cleare, \& Wessely, 2008) participants with such comorbid disorder(s) are usually excluded from the investigations (Baliatsas, Van Kamp, Lebret, \& Rubin, 2012a). This practice leads to excessive sample loss, and, most importantly, sampling bias. Third, as in other areas of medicine, diagnoses based on exclusionary definitions should be avoided. Finally, certain salient aspects of the condition (most importantly, chronicity; M. Witthöft, personal communication) are not included.

The prevalence of IEI-EMF shows a considerable variability (between 1.5-20\%) (Eltiti et al., 2007; Hillert, Berglind, Arnetz, \& Bellander, 2002; Huang, Cheng, \& Guo, 2018; INFAS, 2006; Mohler et al., 2010; Schreier, Huss, \& Röösli, 2006; Ulmer \& Bruse, 2006).This 
variability could be partly explained by the lack of generally accepted medical diagnostic procedure or definition. In fact, more than half of the empirical studies on IEI-EMF applied only participants' self-report about their EMF-hypersensitivity - often assessed using a simple yes-or-no question - as inclusion criterion (Baliatsas et al., 2012b).

Because of the striking similarities between IEIs and medically unexplained symptoms or functional somatic syndromes, many authors suggest that IEIs should be managed as a sub-category of somatoform disorders, where symptoms are attributed to a specific environmental factor (Bailer, Witthöft, Paul, Bayerl, \& Rist, 2005; Henningsen \& Priebe, 2003; Wiesmüller, Ebel, Hornberg, Kwan, \& Friel, 2003). Keeping in mind that IEIEMF is officially often recognized as a functional impairment (Johansson, 2015), and that the WHO definition considers the existence of symptoms and a negative impact on perceived health also essential to the condition, the use of further questions should be warranted from a theoretical point of view. In addition, an overly inclusive criterion can-hinder not only the exploration of the aetiology and the treatment of IEI-EMF patients, but also raises difficulties for the integration of results gained up to the present (Baliatsas et al., 2012a).

The primary goal of the study reported here was the demonstration of heterogeneity within the category of self-reported IEI-EMF. We also attempted to identify self-report questions (items) that are necessary as inclusion criteria.

\section{Method}

\section{Participants}

A non-representative Hungarian community sample was used. Participants $(N=473$; $76.3 \%$ women; age: $35.03 \pm 13.24$ ) were recruited through various groups in the social media that are thematically not connected to environmental intolerances. The study was approved by the research ethics board of the university. Participants received no reward for their participation; all signed an on-line informed consent form before completing the questionnaire on-line.

\section{Questionnaires and Questions}

The questions and the questionnaire were part of a larger study that investigated the connection between environment and health.

Self-diagnosis of IEI-EMF (IEI-EMF) was assessed with a single yes-or-no question ("Many people experience unpleasant symptoms (e.g. headache, nausea, concentration problems, palpitation, etc.) when staying in the vicinity of electromagnetic fields (e.g. near electric devices, computers, electric power lines, or during mobile phone calls). This phenomenon is called electromagnetic hypersensitivity or electrosensitivity. Do you consider yourself to be 
electrosensitive?') (Dömötör, Doering, \& Köteles, 2016; Köteles et al., 2013; Szemerszky, Gubányi, Árvai, Dömötör, \& Köteles, 2015).

Severity of the condition (Impact) was assessed with the following question: "On the whole to what extent do EMF-related symptoms affect your everyday life?" ( $0=$ no impact at all, 1 = some impact, 2 = medium impact, 3 = high impact) (Dömötör et al., 2016;

Dömötör, Szemerszky, \& Köteles, 2019).

Frequency of EMF-related symptoms (Symptoms) was assessed with the following question: "How often do you experience symptoms in the proximity of electric devices?" $(0=$ never, 1 = it happened once, 2 = rarely, 3 = often, 4 = every time) (Dömötör et al., 2019).

The existence of somatic symptoms, regardless of their origin and assumed cause, were assessed with Patient Health Questionnaire Somatic Symptom Severity Scale (PHQ-15) (Kroenke, Spitzer, \& Williams, 2002) which measures the prevalence and severity of 15 common symptoms in a 3-point Likert-scale from 0 ("not bothered at all") to 2 ("bothered a lot"). Higher scores refer to higher prevalence of disturbing symptoms in the past 4 weeks. Scores of 5, 10, and 15 represent cut-off points for low, medium and high somatic symptom severity, respectively (Kroenke et al., 2002). In clinical practice, PHQ-15 is often used to measure somatization tendency. The Cronbach's alpha coefficient of the scale in the present study was 0.80 .

\section{Statistical Analysis}

Statistical analysis was carried out with the SPSS v20 software. According to the results of Shapiro-Wilk tests, PHQ-15 scores showed a significant deviation from normal distribution, thus non-parametric methods were used throughout the analysis. Groups with and without IEI-EMF were compared using Mann-Whitney-U-tests and chi-square tests (for gender ratio).

\section{Results}

Overall, $15.2 \%$ of the individuals (72 participants) labelled themselves as being hypersensitive to EMF (IEI-EMF item). Descriptive statistics and the results of group-level comparisons are presented in Table 1.

Mann-Whitney-U-tests indicated a significant difference between the self-reported IEI-EMF and non-IEI-EMF group in PHQ-15 score, frequency of IEI-EMF related symptoms, and impact of EMF-related symptoms on everyday life. The IEI-EMF group was characterized by higher values in all cases, and it approached but did not reach the PHQ-15 cut-off point for medium symptom severity. There was no significant difference between the two groups with respect to gender ratio and age. 
Table 1

Descriptive Statistics of the Measured Variables (Mean \pm Standard Deviation)

\begin{tabular}{|c|c|c|c|c|}
\hline \multirow[b]{2}{*}{ Variable } & \multirow{2}{*}{$\begin{array}{l}\text { IEI-EMF } \\
(N=72)\end{array}$} & \multirow{2}{*}{$\begin{array}{l}\text { non-IEI-EMF } \\
(N=401)\end{array}$} & \multicolumn{2}{|c|}{ Between-group comparison } \\
\hline & & & Statistic & $p$ \\
\hline Age & $36.0 \pm 13.54$ & $34.86 \pm 13.20$ & $\mathrm{M}-\mathrm{W} \mathrm{U}=13579.50$ & $>.05$ \\
\hline Gender ratio (women) & $82 \%$ & $75 \%$ & $\chi^{2}=1.49$ & $>.05$ \\
\hline Somatic symptoms (PHQ-15) & $9.17 \pm 4.67$ & $7.37 \pm 4.47$ & $M-W U=10916.50$ & .001 \\
\hline Impact on daily life & $0.74 \pm 0.69$ & $0.1 \pm 0.33$ & $\mathrm{M}-\mathrm{W} \mathrm{U}=6894.50$ & $<.001$ \\
\hline Frequency of symptoms & $2.0 \pm 1.08$ & $0.4 \pm 0.78$ & $\mathrm{M}-\mathrm{W} \mathrm{U}=4328.50$ & .001 \\
\hline
\end{tabular}

Note. IEI-EMF = Idiopathic Environmental Intolerance attributed to Electromagnetic Fields; PHQ-15 = Patient Health Questionnaire; M-W U = Mann-Whitney $U$.

If we apply another inclusion criterion, i.e., the rare (but already regular) occurrence of symptoms (Symptoms $>1$ ), altogether $25.37 \%$ of the 473 participants (120 individuals) reported that they had experienced symptoms attributed to electromagnetic field exposure at least occasionally. Of these 120 individuals, however, only $47.5 \%$ (57 individuals) considered themselves electrohypersensitive, whereas 63 did not. Both groups' PHQ-15 score was below the cut-off point (IEI-EMF: $9.30 \pm 4.40$; non-IEI-EMF: $8.54 \pm 4.84$ ), and showed no significant difference (Mann-Whitney $U=1586.00, p=.269$ ).

Similarly, considering a minimal impact of the condition on everyday functioning (Impact $>0$ ), it turns out that 82 individuals of the 473 participants $(17.34 \%$ ) belong to this category. Interestingly, only 53.7\% (44 individuals) diagnosed themselves as IEI-EMF, while the remaining $38 \mathrm{did}$ not use this label. In both groups, the PHQ-15 score exceeds the medium cut-off point (IEI-EMF: $10.02 \pm 4.65$; non-IEI-EMF: $10.13 \pm 4.88$ ), but they did not differ from each other (Mann-Whitney $U=831.50, p=.967$ ).

Taken together, only 44 of the 72 individuals $(61.1 \%)$ with self-reported IEI-EMF had symptoms attributed to electromagnetic devices at least rarely and suffered from the condition at least slightly. Surprisingly, there were 15 individuals (20.8\%) who had neither symptoms nor a negative impact on their everyday functioning but still considered themselves IEI-EMF. In the non-IEI-EMF group, 25 individuals (6.2\%) were characterized by both criteria (for details, see Figure 1). Within those, who reported symptoms and also an impact on daily life (69 individuals), the IEI-EMF group's PHQ-15 score was slightly above the cut-off point (10.02 \pm 4.65$)$, while the non-IEI-EMF group scored a bit lower (9.76 \pm 4.94$)$. Still, the difference between the two was not significant (Mann-Whitney $U=523.00, p=.735)$. Finally, average PHQ-15 score of the group defined by symptoms and impact regardless of the IEI-EMF label was close to the threshold of $10(9.93 \pm 4.72)$. 


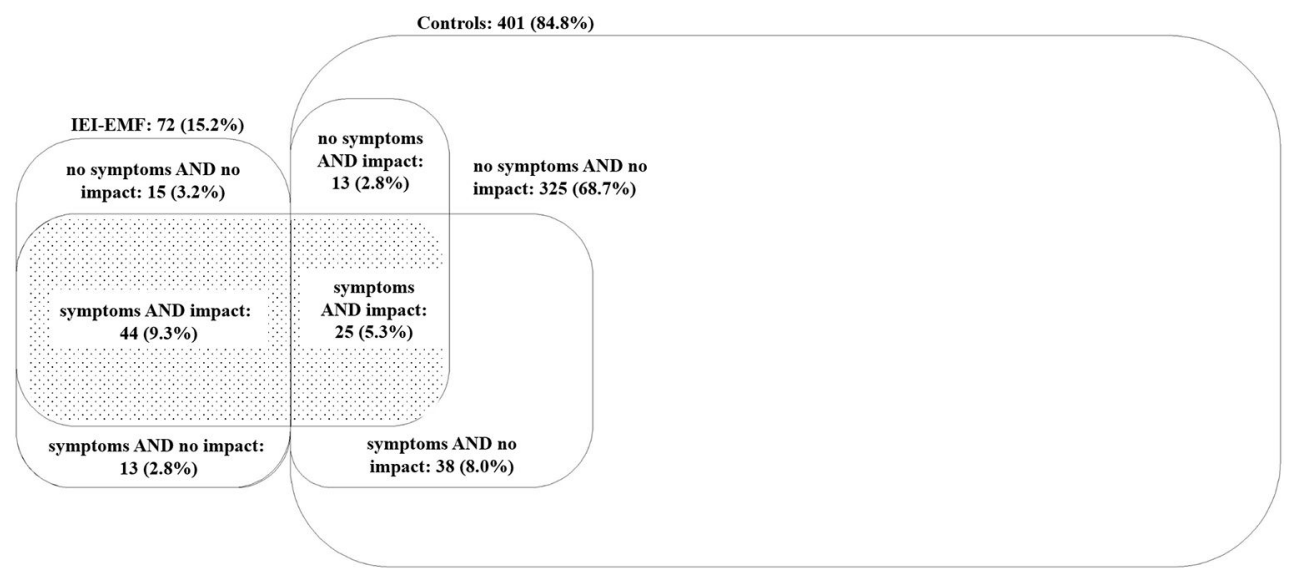

Figure 1. The number of individuals (and their percentage of total) in the IEI-EMF and control group after applying additional inclusion criteria beyond self-reported electrohypersensitivity.

\section{Discussion}

Our results demonstrate that the use of one single yes-or-no question as inclusion criterion for self-reported IEI-EMF is not an acceptable practice. Although the IEI-EMF group selected by this single question shows a higher average somatization tendency than the non-IEI-EMF group, this tendency is still under the accepted threshold of medium impact.

Beyond the widely applied yes-or-no question, the use of at least two additional questions appears to be necessary for a more precise definition of the condition and the sample. After the use of three inclusion criteria instead of one, only $61 \%$ of the individuals of the original IEI-EMF group remained there. The two additional criteria, i.e., experiencing symptoms attributed to EMF on a regular basis and symptoms impacting everyday functioning, are in accordance with the WHO definition of the condition. Moreover, this more strictly (still rather inclusively) defined group shows a score that indicates a non-negligible somatization tendency. This latter finding is in line with the conceptualization of IEI as a sub-category of functional somatic syndromes (Frick, Rehm, \& Eichhammer, 2002).

In fact, self-diagnosis (i.e. the acceptance of the IEI-EMF label) is not part of the WHO definition thus the use of the yes-or-no question can be questioned. In our sample, 69 individuals of the $473(14.6 \%)$ belong to the IEI-EMF group as defined by the symptoms and impact question. Somatization tendency of this group practically reached the threshold of medium severity, and applying the IEI-EMF label did not elevate this score substantially. In other words, using the criteria of symptoms and impact appears to be practically sufficient as well as in line with the definition of the condition. 
Beyond practical issues, the present findings demonstrate that individuals with selfdiagnosed IEI-EMF does not represent a homogeneous group. It is particularly striking that $21 \%$ of the individuals labelling themselves as IEI-EMF experience neither symptoms nor any negative impact on their daily life. This finding can be explained by two approaches. First, stories about harmful effects of modern technologies are abundant in mass media and impact not only people's worrying tendency (Bräscher, Raymaekers, Van den Bergh, \& Witthöft, 2017; Petrie et al., 2001; Witthöft et al., 2018), but also their automatic self-perception and self-categorization. Second, as in the case of complementary and alternative medicine (Astin, 1998), philosophical congruence might be a motive for those characterized by an experiential-intuitive thinking style to accept the IEI-EMF label, even in the absence of symptoms.

The most important limitation of the present study is that our sample was not representative of the population, therefore the results are not generalizable. Additionally, the applied sampling method (online assessment) has well-known limitations. Finally, identification of people suffering from IEI-EMF based only self-report questions without any external criterion or assessment could be equivocal.

In summary, a minimum of two questions appear to be sufficient as inclusion criteria for IEI-EMF in empirical research. Instead of the widely used yes-or-no question on accepting the IEI-EMF label, regular occurence of symptoms attributed to EMF and at least a slight negative impact on daily life are required.

Funding: The authors received no financial support for the research, authorship, and/or publication of this article.

Competing Interests: The authors declare no conflicts of interest.

Acknowledgments: This research was supported by the Hungarian National Scientific Research Fund (K 124132), the János Bolyai Research Scholarship of the Hungarian Academy of Sciences (for R. Szemerszky) and by the ÚNKP-17-3 New National Excellence Program of the Ministry of Human Capacities (for Zs. Dömötör).

\section{References}

Astin, J. A. (1998). Why patients use alternative medicine. FAMA: The fournal of the American Medical Association, 279(19), 1548-1553. https://doi.org/10.1001/jama.279.19.1548

Bailer, J., Witthöft, M., Paul, C., Bayerl, C., \& Rist, F. (2005). Evidence for overlap between idiopathic environmental intolerance and somatoform disorders. Psychosomatic Medicine, 67(6), 921-929. https://doi.org/10.1097/01.psy.0000174170.66109.b7

Baliatsas, C., Van Kamp, I., Lebret, E., \& Rubin, G. J. (2012a). Idiopathic environmental intolerance attributed to electromagnetic fields (IEI-EMF): A systematic review of identifying criteria. $B M C$ Public Health, 12(1), Article 643. https://doi.org/10.1186/1471-2458-12-643 
Baliatsas, C., Van Kamp, I., Bolte, J., Schipper, M., Yzermans, J., \& Lebret, E. (2012b). Non-specific physical symptoms and electromagnetic field exposure in the general population: Can we get more specific? A systematic review. Environment International, 41, 15-28.

https://doi.org/10.1016/j.envint.2011.12.002

Berg, N. D., Rasmussen, H. B., Linneberg, A., Brasch-Andersen, C., Fenger, M., Dirksen, A., . . Elberling, J. (2010). Genetic susceptibility factors for multiple chemical sensitivity revisited. International fournal of Hygiene and Environmental Health, 213(2), 131-139. https://doi.org/10.1016/j.ijheh.2010.02.001

Bräscher, A.-K., Raymaekers, K., Van den Bergh, O., \& Witthöft, M. (2017). Are media reports able to cause somatic symptoms attributed to WiFi radiation? An experimental test of the negative expectation hypothesis. Environmental Research, 156, 265-271.

https://doi.org/10.1016/j.envres.2017.03.040

Caccamo, D., Cesareo, E., Mariani, S., Raskovic, D., Ientile, R., Currò, M., . . De Luca, C. (2013). Xenobiotic sensor- and metabolism-related gene variants in environmental sensitivity-related illnesses: A survey on the Italian population. Oxidative Medicine and Cellular Longevity, 2013, Article 831969. https://doi.org/10.1155/2013/831969

Cui, X., Lu, X., Hiura, M., Oda, M., Miyazaki, W., \& Katoh, T. (2013). Evaluation of genetic polymorphisms in patients with multiple chemical sensitivity. PloS One, 8(8), Article e73708. https://doi.org/10.1371/journal.pone.0073708

De Luca, C., Gugliandolo, A., Calabrò, C., Currò, M., Ientile, R., Raskovic, D., . . Caccamo, D. (2015). Role of polymorphisms of inducible nitric oxide synthase and endothelial nitric oxide synthase in idiopathic environmental intolerances. Mediators of Inflammation, 2015, Article 245308. https://doi.org/10.1155/2015/245308

De Luca, C., Thai, J. C. S., Raskovic, D., Cesareo, E., Caccamo, D., Trukhanov, A., \& Korkina, L. (2014). Metabolic and genetic screening of electromagnetic hypersensitive subjects as a feasible tool for diagnostics and intervention. Mediators of Inflammation, 2014, Article 924184. https://doi.org/10.1155/2014/924184

Dömötör, Z., Doering, B. K., \& Köteles, F. (2016). Dispositional aspects of body focus and idiopathic environmental intolerance attributed to electromagnetic fields (IEI-EMF). Scandinavian fournal of Psychology, 57(2), 136-143. https://doi.org/10.1111/sjop.12271

Dömötör, Z., Szemerszky, R., \& Köteles, F. (2019). Nature relatedness is connected with modern health worries and electromagnetic hypersensitivity. Journal of Health Psychology, 24(12), 1756-1764. https://doi.org/10.1177/1359105317699681

Eltiti, S., Wallace, D., Zougkou, K., Russo, R., Joseph, S., Rasor, P., \& Fox, E. (2007). Development and evaluation of the electromagnetic hypersensitivity questionnaire. Bioelectromagnetics, 28(2), 137-151. https://doi.org/10.1002/bem.20279

Frick, U., Kharraz, A., Hauser, S., Wiegand, R., Rehm, J., von Kovatsits, U., \& Eichhammer, P. (2005). Comparison perception of singular transcranial magnetic stimuli by subjectively electrosensitive subjects and general population controls. Bioelectromagnetics, 26(4), 287-298. https://doi.org/10.1002/bem.20085 
Frick, U., Rehm, J., \& Eichhammer, P. (2002). Risk perception, somatization, and self report of complaints related to electromagnetic fields - A randomized survey study. International fournal of Hygiene and Environmental Health, 205(5), 353-360. https://doi.org/10.1078/1438-4639-00170

Henningsen, P., \& Priebe, S. (2003). New environmental illnesses: What are their characteristics? Psychotherapy and Psychosomatics, 72(5), 231-234. https://doi.org/10.1159/000071893

Hillert, L., Berglind, N., Arnetz, B. B., \& Bellander, T. (2002). Prevalence of self-reported hypersensitivity to electric or magnetic fields in a population-based questionnaire survey. Scandinavian fournal of Work, Environment \& Health, 28(1), 33-41.

https://doi.org/10.5271/sjweh.644

Huang, P.-C., Cheng, M.-T., \& Guo, H.-R. (2018). Representative survey on idiopathic environmental intolerance attributed to electromagnetic fields in Taiwan and comparison with the international literature. Environmental Health, 17, Article 5.

https://doi.org/10.1186/s12940-018-0351-8

INFAS. (2006). Ermittlung der Befürchtungen und Ängste der breiten Öffentlichkeit hinsichtlich möglicher Gefahren der hochfrequenten elektromagnetischen Felder des Mobilfunks: Abschlussbericht über die Befragung im fahr 2006. Bonn, Germany: Institut für Angewandte Sozialwissenschaft.

Johansson, O. (2015). Electrohypersensitivity: A functional impairment due to an inaccessible environment. Reviews on Environmental Health, 30(4), 311-321. https://doi.org/10.1515/reveh-2015-0018

Köteles, F., Szemerszky, R., Gubányi, M., Körmendi, J., Szekrényesi, C., Lloyd, R., . . Bárdos, G. (2013). Idiopathic environmental intolerance attributed to electromagnetic fields (IEI-EMF) and electrosensibility (ES) - Are they connected? International fournal of Hygiene and Environmental Health, 216(3), 362-370. https://doi.org/10.1016/j.ijheh.2012.05.007

Kroenke, K., Spitzer, R. L., \& Williams, J. B. W. (2002). The PHQ-15: Validity of a new measure for evaluating the severity of somatic symptoms. Psychosomatic Medicine, 64(2), 258-266. https://doi.org/10.1097/00006842-200203000-00008

Landgrebe, M., Barta, W., Rosengarth, K., Frick, U., Hauser, S., Langguth, B., . . Eichhammer, P. (2008). Neuronal correlates of symptom formation in functional somatic syndromes: A fMRI study. NeuroImage, 41(4), 1336-1344. https://doi.org/10.1016/j.neuroimage.2008.04.171

Lessof, M. (1997). Report of Multiple Chemical Sensitivities (MCS) Workshop, Berlin, Germany, 21-23 February 1996. PCS/96.29 IPCS, Geneva, Switzerland. Human \& Experimental Toxicology, 16(4), 233-234. https://doi.org/10.1177/096032719701600414

McKeown-Eyssen, G., Baines, C., Cole, D. E. C., Riley, N., Tyndale, R. F., Marshall, L., \& Jazmaji, V. (2004). Case-control study of genotypes in multiple chemical sensitivity: CYP2D6, NAT1, NAT2, PON1, PON2 and MTHFR. International fournal of Epidemiology, 33(5), 971-978. https://doi.org/10.1093/ije/dyh251

Meg Tseng, M.-C., Lin, Y.-P., \& Cheng, T.-J. (2011). Prevalence and psychiatric comorbidity of selfreported electromagnetic field sensitivity in Taiwan: A population-based study. Fournal of the Formosan Medical Association, 110(10), 634-641. https://doi.org/10.1016/j.jfma.2011.08.005 
Mohler, E., Frei, P., Braun-Fahrländer, C., Fröhlich, J., Neubauer, G., \& Röösli, M. (2010). Effects of everyday radiofrequency electromagnetic-field exposure on sleep quality: A cross-sectional study. Radiation Research, 174(3), 347-356. https://doi.org/10.1667/RR2153.1

Österberg, K., Persson, R., Karlson, B., Eek, F. C., \& Ørbæk, P. (2007). Personality, mental distress, and subjective health complaints among persons with environmental annoyance. Human \& Experimental Toxicology, 26(3), 231-241. https://doi.org/10.1177/0960327107070575

Petrie, K. J., Sivertsen, B., Hysing, M., Broadbent, E., Moss-Morris, R., Eriksen, H. R., \& Ursin, H. (2001). Thoroughly modern worries: The relationship of worries about modernity to reported symptoms, health and medical care utilization. fournal of Psychosomatic Research, 51(1), 395-401. https://doi.org/10.1016/S0022-3999(01)00219-7

Rubin, G. J., Cleare, A. J., \& Wessely, S. (2008). Psychological factors associated with self-reported sensitivity to mobile phones. Fournal of Psychosomatic Research, 64(1), 1-9. https://doi.org/10.1016/j.jpsychores.2007.05.006

Schnakenberg, E., Fabig, K.-R., Stanulla, M., Strobl, N., Lustig, M., Fabig, N., \& Schloot, W. (2007). A cross-sectional study of self-reported chemical-related sensitivity is associated with gene variants of drug-metabolizing enzymes. Environmental Health, 6, Article 6. https://doi.org/10.1186/1476-069X-6-6

Schreier, N., Huss, A., \& Röösli, M. (2006). The prevalence of symptoms attributed to electromagnetic field exposure: A cross-sectional representative survey in Switzerland. SozialUnd Präventivmedizin, 51(4), 202-209. https://doi.org/10.1007/s00038-006-5061-2

Staudenmayer, H. (2006). Idiopathic Environmental Intolerance (IEI): A causation analysis. In K. Hansson Mild, M. Repacholi, E. van Deventer, \& P. Ravazzani (Eds.), Electromagnetic Hypersensitivity. Proceedings: International Workshop on EMF Hypersensitivity (pp. 39-53). Prague, Czech Republic: WHO.

Szemerszky, R., Gubányi, M., Árvai, D., Dömötör, Z., \& Köteles, F. (2015). Is there a connection between electrosensitivity and electrosensibility? A replication study. International fournal of Behavioral Medicine, 22(6), 755-763. https://doi.org/10.1007/s12529-015-9477-z

Ulmer, S., \& Bruse, M. (2006). Supplementary Information on Electromagnetic Hyopersensitive. Final report German Mobile Telecommunication Research Program. Retrieved from http://www.emf-forschungsprogramm.de

WHO. (2004). WHO workshop on electrical hypersensitivity - Working group meeting report. Prague, Czech Republic: Author.

Wiesmüller, G. A., Ebel, H., Hornberg, C., Kwan, O., \& Friel, J. (2003). Are syndromes in environmental medicine variants of somatoform disorders? Medical Hypotheses, 61(4), 419-430. https://doi.org/10.1016/S0306-9877(03)00185-3

Witthöft, M., Freitag, I., Nußbaum, C., Bräscher, A.-K., Jasper, F., Bailer, J., \& Rubin, G. J. (2018). On the origin of worries about modern health hazards: Experimental evidence for a conjoint influence of media reports and personality traits. Psychology \& Health, 33(3), 361-380. https://doi.org/10.1080/08870446.2017.1357814 


\section{EACLIPT}

Clinical Psychology in Europe (CPE) is the official journal of the European Association of Clinical Psychology and Psychological Treatment (EACLIPT).

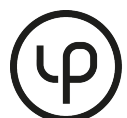

leibniz-psychology.org

PsychOpen GOLD is a publishing service by Leibniz Institute for Psychology Information (ZPID), Germany. 